\title{
The effect of paint types toward iris color changes of ocular prosthetics
}

\author{
Gunawan Sri Sarjono*, Titik Ismiyati**, Endang Wahyuningtyas**® \\ *Prosthodontics Specialty Program, Faculty of Dentistry, Universitas Gadjah Mada, Yogyakarta, Indonesia \\ **Department of Prosthodontics, Faculty of Dentistry, Universitas Gadjah Mada, Yogyakarta, Indonesia \\ **JI Denta No 1, Sekip Utara, Yogyakarta, Indonesia; $₫$ correspondence: endang_wtyas2014@ugm.ac.id
}

Submitted: $11^{\text {st }}$ September 2018; Revised: $4^{\text {th }}$ April 2019; Accepted: $24^{\text {th }}$ June 2019

\begin{abstract}
Ocular prosthetics are mainly created to meet the aspect of esthetic consideration. The paint application in ocular prosthetics construction is remaining an option for Prosthodontist. This study aims to examine the effect of types of paint toward iris color change of the ocular prosthetics. Three black types of paint: oil paint (Maries Oil Color, China), automotive paint (Avian, Indonesia), and acrylic paint (Sakura, Japan) were used to paint paper disc to produce 27 iris ocular prosthetics. Chromameter was used to examine the first and the final color after acrylic processing using the microwave. The measurement results in the process using Commission Internationale de l'Eclairage. One-way ANOVA test showed significant differences between the three types of paints on ocular prosthetics $(p<0.05)$. The LSD test revealed that oil paint groups had more significant results than the automotive paint group and acrylic paint group, while the automotive paint group did not have significant differences with the acrylic paint group ( $p>0.05)$. Thus, it is conclusive that oil paint influences the iris color change of the ocular prosthetics.
\end{abstract}

Keywords: iris color change; ocular prosthetics; types of paint

\section{INTRODUCTION}

Eye loss does not only lead a person to experience a functional disability but also affects one's aesthetic appearance. ${ }^{1}$ The creation of ocular prosthetics involves a sensitive technical procedure to ensure accurate duplication of the color, size, contour, and orientation of the natural eye as expected by the patient. ${ }^{2,3}$ During iris coloring, the technique of making and selecting the right type of paint is essential. The determination stage of the right iris color is the hardest part because of the stability of the paint color cannot be controlled during the acrylic polymerization process. ${ }^{3}$

Color changes in the manufacture of ocular prosthetics may occur due to the interaction between the paint and acrylic resin components because the presence of residual acrylic monomers affects the paint component, which is also a polymer. The determination stage of right iris color is complicated because the paint color stability cannot be controlled during the acrylic polymerization process. ${ }^{3,4}$
Therefore, during the coloring process, it is necessary to determine the correct type of paint and technique. Several studies have been conducted to find the appropriate color formation and the most suitable method in iris coloring. Research using oil paint, watercolors, acrylic paints, and automotive paints in brown and blue iris coloring concluded that blue oil paint had the best stability compared to other types of paint. ${ }^{5}$ On this basis, it is necessary to study the effect of type of paints on the iris color in black eye prostheses given the fact there was no research to address this issue.

\section{MATERIALS AND METHODS}

This study has been approved to meet the ethical aspect based on the ethical clearance number 001137/KKEP/FKG-UGM/EC/2017 from ethic research Committee Faculty of Dentistry Universitas Gadjah Mada. The study used twenty-seven samples of hot polymerized clear acrylic discs white acrylic (GC, China) samples, and waterproof papers 
with a diameter of $15 \mathrm{~mm}$ and $1 \mathrm{~mm}$ thickness divided into three groups. The first group consisted of nine samples of clear acrylic plates, colored with black colored acrylic paint. The second group comprised of nine samples of clear acrylic plates stained with black oil paint. The last consisted of nine samples of clear acrylic plates stained with black automotive paint. The measurement of the initial color of the sample on acrylic plates was done using chromameter. After initial measurements, samples were heated with low power of 90 watts for 13 minutes, 1 minute 12 seconds with a high power of 500 watts in microwave and were allowed to cool for 30 minutes. Color change measurements were carried out after treatment using chromameter (IKA, USA) for all samples according to the method of the commission of Internationale de l'Eclairage. ${ }^{1}$ The color change data were analyzed by one-way ANOVA. Previously, the normality of the data was tested using Shapiro Wilk and the homogeneity of the data was tested with Levene's test.

\section{RESULTS}

The color change of the sample type of paint is presented in Table 1, Figure 1 and Figure 2. The lowest mean of iris staining changes is shown by the oil paint group $(0.712 \pm 0.081)$. The highest average coloring change is indicated by the automotive paint group $(2.807 \pm 0.825)$.

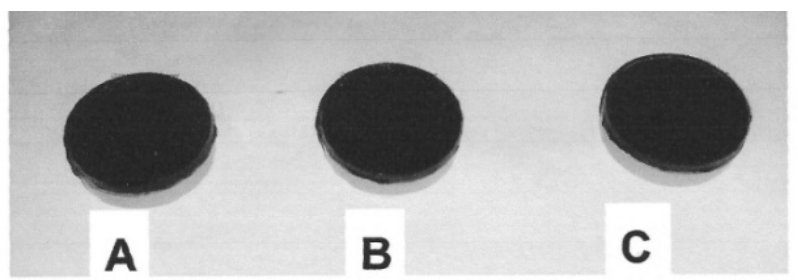

Figure1. The sample of automotive paint $(A)$, oil paint $(B)$, acrylic paint $(C)$, before heated in the microwave

Table 1. Average and standard deviation of iris color changes in ocular prosthetics with different types of paint

\begin{tabular}{lcc}
\hline Treatment group & $\begin{array}{c}\text { Number of } \\
\text { samples }\end{array}$ & Average \pm SD \\
\hline Automotive paints & 9 & $2.807 \pm 0.825$ \\
Oil paints & 9 & $0.712 \pm 0.081$ \\
Acrylic paints & 9 & $2.253 \pm 0.766$ \\
\hline
\end{tabular}

SD: Standard deviation

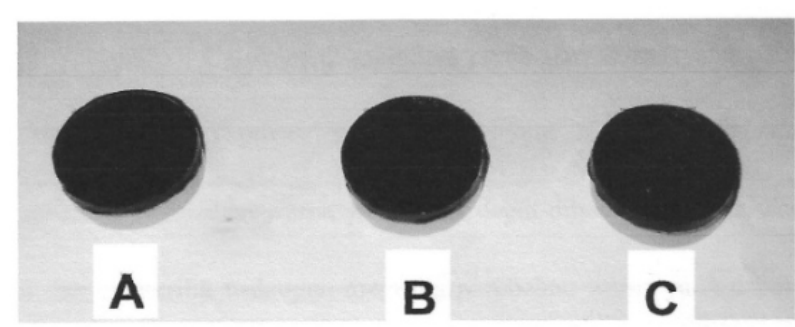

Figure 2. The sample of automotive paint $(A)$, oil paint $(B)$, acrylic paint $(C)$, after heated in the microwave

Table 2. Results of normality changes to iris color changes in eye prosthetics with different types of paint

\begin{tabular}{lcc}
\hline \multicolumn{3}{c}{ Shapiro-Wilk test } \\
\hline \multicolumn{1}{c}{ Groups } & Statistics & Probability $(p)$ \\
\hline Automotive paints & 0.891 & 0.203 \\
Oil paints & 0.889 & 0.195 \\
Acrylic paints & 0.941 & 0.589 \\
\hline
\end{tabular}

The normality test with Shapiro-Wilk in Table 2 presents that the data of iris coloring changes in ocular prosthetics for all types of paints has a $p$ value of $>0.05$. Therefore, the data population had a normal distribution. Levene's test results in iris color changes of different types of ocular prosthetics using different types of paint showed $p=$ 0.7 ( $p>0.05$ ). In other words, the population variant is homogeneous. One-way ANOVA test results revealed significant differences between different types of paints in ocular prosthetic $(p<0.05)$.

The LSD test result in Table 3 showed the differences in iris staining changes. Thus, it is possible to draw the following conclusions: the oil paint group showed the most significant difference $(p<0.05)$ of other types of paint, while the automotive paint group did not lead to a significant difference as compared to the acrylic paint group ( $p>0.05)$.

Table 3. Least significances difference (LSD) test result of iris color changes in ocular prosthetic with different types of paint

\begin{tabular}{lccc}
\hline Type of paint & Oil paint & $\begin{array}{c}\text { Acrylic } \\
\text { paint }\end{array}$ & $\begin{array}{c}\text { Automotive } \\
\text { paint }\end{array}$ \\
\hline $\begin{array}{l}\text { Oil paint } \\
\text { Acrylic paint }\end{array}$ & $-1.540^{*}$ & & $-2.0944^{*}$ \\
Automotive paint & & 0.5542 & \\
\hline
\end{tabular}

*: There are significant differences 
Majalah Kedokteran Gigi Indonesia. April 2020; 6(1): 60 - 63

ISSN 2460-0164 (print)

ISSN 2442-2576 (online)

\section{DISCUSSION}

The results of the measurement of color changes in paint used in making eye iris prosthesis showed a variation in mean values. The smallest mean value of color change is resulted by the oil paint. Oil paint has better color stability due to the content of opacifier (zinc oxide) making it more resistant to temperature changes. ${ }^{4,6}$ Moreover, it contains an additive material, which causes more resistance to polymerization heating of acrylic and microwave heating. Automotive paint has the highest average color change of all the three because the content of binder in the form of acrylic resin in automotive paint causes instability when chemical reactions occur with acrylic monomers and electromagnetic waves generated by microwaves. ${ }^{7}$ Automotive paints and acrylic paints have the same binder, acrylic resin. Heat damages the binder and it causes color changes. ${ }^{6}$ Research on the blue color revealed that oil paint has the lowest color changes of all other three (watercolor, automotive paint, and acrylic paint) ${ }^{8}$

One-way ANOVA test demonstrated that there were significant different results between oil paint, acrylic paint, and automotive paint $(p<0.05)$. The three types of paint underwent a process of discoloration. This process made the oil paint have the smallest color change, followed by acrylic paint, and automotive paint. This result is attributed to the fact that the type of oil paint solvent uses natural oil, known as flaxseed oil which is more resistant to temperature changes during polymerization. ${ }^{8}$ The oil paint binding material does not contain water so that it does not experience any evaporation in the drying process, but the reaction absorbs oxygen from the air. When the iris was heated in the microwave, there was no excessive evaporation of water from the oil paint. This is what makes it different from acrylic paints and automotive paints which have water-based solvents. The water-based solvents in the acrylic and automotive paints led to the drying process that involved water evaporation to remove the paint component. Normally, the drying process of acrylic and automotive paint can take days to a year. The exposure to heat during microwave heating fastened the water evaporation of the paint, damaging the chemical bond in the paint. $^{7}$

The LSD test in iris changes staining in ocular prosthetics with different types of coloring paint showed a significant difference between oil paint, automotive paint, and acrylic $(p<0.05)$. However, the LSD test of automotive paint and acrylic paint did not differ significantly $(p>0.05)$. The temperature of heat produced during the polymerization process of acrylic and heating using a microwave can affect the color used in making iris. Temperature is an important factor in the degeneration process (oxidation or hydrolysis) since it can damage the polymer bonds. ${ }^{7,9}$ The research by Bonnati concluded that the color change of less than 1.00 cannot be seen visually, but the color changes of less than 3.00 can be accepted clinically. ${ }^{10}$ The process of damage to these chemical bonds can also be observed in the effects of long-term use of paint exposed to physical and chemical conditions, such as exposure to changes in temperature and humidity. ${ }^{11}$ The results of acrylic paints and automotive paints have an insignificant differences compared to the result of oil paints because they have the same binder composition of acrylic resin. ${ }^{12}$

Changes of less than 1.00 cannot be observed visually, while changes of less than 3.3 are still clinically acceptable. However, the changes of more than 3.3 of these materials are not suitable for use..$^{13}$ This means that clinical discoloration and the results obtained from this study are still acceptable (the biggest average is automotive paint with 2.807 \pm 0.825 ). Oil paint has the best color change because after going through a further polymerization process using a microwave, its color changes could not be distinguished visually. ${ }^{14,15}$ Although the discoloration of automotive paints and acrylic paints was visible, the results were still clinically acceptable. Thus, it indicates that this type of paint is influential in the manufacture of ocular prosthetics. Oil paint has the smallest color change of all the three types of paints. Clinically, the changes in the three types of paint are still acceptable, but the changes experienced by oil paint cannot be distinguished visually. 


\section{CONCLUSION}

Based on the study, it is concluded that the type of oil paint affects the iris color changes in the ocular prosthesis. Further research needs to determine the effect of the type of paint used for eye prosthesis by considering the sunlight factor of the color changes in the ocular prosthetics.

\section{REFERENCES}

1. Artopolou I, Montgomery P, Wesly P, Lemon $J$. Digital imaging in fabriccation of ocular prosthetics. J Prosthet Dent. 2006; 95: 327-330.

doi: 10.1016/j.prosdent.2006.01.018

2. Bali N, Dhall RS, Singh N. Various step involved in fabrication of an ocular prosthetic: a case report. Int J Dent Med Res. 2015; 1(5): 93-96.

3. Atay A. Overview of maxillofacial prosthetics. New York: Nova Sciences Publishers, Inc.; 2013. 60-64.

4. Goiato M. Santos D, Moreno A, Gennari-Filho $\mathrm{H}$, Pellizzer E. Evaluation of color stability of two technicueqes for reproducing artificial iriades after microwave polymeritation. J Appl Oral Sci. 2011; 19(3): 200-203. doi: 10.1590/S1678-77572011000300004

5. Reis RC, Dias R, Carvalho J. Evaluation of iris color stability in ocular prosthetics. Braze Dent J. 2008; 19(4): 370-374. doi: 10.1590/s0103-64402008000400015
6. Assuncao WG. Effect of polimerization method and thermal cycling on color stability of acrylic resin denture teeth. J Prosthet Dent. 2009; 102(6): 385-392. doi: 10.1016/S0022-3913(09)60200-6

7. Goiato MC, Santos D, Moreno A, GennariFilho $\mathrm{H}$, Pellizzer E. Evaluation of the color stability of two technicueqes for reproducing artificial irides after microwave polymeritation. J Appl Oral Sci. 2011; 19(3): 200-203. doi: 10.1590/S1678-77572011000300004

8. Fernandes A, Goiato M, Batista M, Santos D. Color alteration of the paint used for iris painting in ocular prostheses. Braz Oral Res. 2008; 23(4): 386-392.

9. Bannwart LC, Goiato MC, dos Santos DM, Moreno A, Pesqueira AA, Haddad MF, Andreotti AM, de Medeiros RA. Chromatic changes to artificial irises produced using different techniques. J Biomed Opt. 2013; 18(5): 82-89. doi: 10.1117/1.JBO.18.5.058002

10. Bonnati M, Chunca T, Regis R, Silva-Lopato $\mathrm{C}$, Paranhos $\mathrm{H}$. The effect of polimerization cycles on color stability on microwaveprocessed denture base resin. J Prosthet. dent. 2009; 18(5): 432-437. doi: 10.1111/j.1532-849X.2009.00458.

11. Ahmad N, Verma A, Ali M, Srivastava M. Prosthetic rehabilitation of ocular defect with customized iris, a case report. MDJ. 2014; 36(2): $1-5$. 